\title{
Synthetic long oligonucleotides to generate artificial templates for use as positive controls in molecular assays: drug resistance mutations in influenza virus as an example
}

\author{
Bin Wang ${ }^{1 *}$, Megan C Steain ${ }^{1,2}$, Dominic E Dwyer ${ }^{1,3}$, Anthony L Cunningham ${ }^{1}$ and Nitin K Saksena ${ }^{1}$
}

\begin{abstract}
Background: Positive controls are an integral component of any sensitive molecular diagnostic tool, but this can be affected, if several mutations are being screened in a scenario of a pandemic or newly emerging disease where it can be difficult to acquire all the necessary positive controls from the host. This work describes the development of a synthetic oligo-cassette for positive controls for accurate and highly sensitive diagnosis of several mutations relevant to influenza virus drug resistance.

Results: Using influenza antiviral drug resistance mutations as an example by employing the utility of synthetic paired long oligonucleotides containing complementary sequences at their $3^{\prime}$ ends and utilizing the formation of oligonucleotide dimers and DNA polymerization, we generated $~ 170 \mathrm{bp}$ dsDNA containing several known specific neuraminidase inhibitor (NAl) resistance mutations. These templates were further cloned and successfully applied as positive controls in downstream assays.
\end{abstract}

Conclusion: This approach significantly improved the development of diagnosis of resistance mutations in terms of time, accuracy, efficiency and sensitivity, which are paramount to monitoring the emergence and spread of antiviral drug resistant influenza strains. Thus, this may have a significantly broader application in molecular diagnostics along with its application in rapid molecular testing of all relevant mutations in an event of pandemic.

\section{Background}

Recently, the occurrence in humans of infection with virulent avian influenza $\mathrm{A}$ H5N1 and the emergence of swine origin pandemic influenza A H1N1 2009 strain have sparked fear of an ongoing pandemic with novel genetic characters [1-3]. While vaccines remain the most effective public health strategy for prevention $[4,5]$, antiviral drugs such as neuraminidase inhibitors (NAIs), oseltamivir and zanamivir, could play an important role in the response to the early phases of a pandemic, if available in sufficient quantities. However, like other antiviral agents, the emergence of influenza viruses with reduced susceptibility to the NAI is inevitable during

\footnotetext{
* Correspondence: bin.wang@usyd.edu.au

'Retroviral Genetics Laboratory, Centre for Virus Research, Westmead Millennium Institute, Westmead Hospital, The University of Sydney,

Westmead, New South Wales 2145, Australia

Full list of author information is available at the end of the article
}

treatment [1]. To date, strains with altered susceptibility to NAI have been recovered from approximately $1 \%$ of immunocompetent adult patients [6] and up to $18 \%$ of pediatric patients [7]. In addition, oseltamivir-resistant influenza A H5N1 and pandemic H1N1 2009 viruses with the H274Y mutation have been reported from patients during oseltamivir treatment $[8,9]$.

Significant advances in molecular biology and human genomic research has paved the way for a host of new genetic diagnostic tests, including gene sequencing, detection, identification and genotyping of organisms using real time polymerase chain reaction (PCR) or other amplification techniques such as multiplex PCR, reverse line blot hybridization (RLB) [10], Ligase chain reaction (LCR), Rolling Circle Amplification (RCA) $[9,11]$, microarray [12-14]. Sequencing often serves as a 'gold standard' for the detection of single nucleotide polymorphisms, drug resistance mutations or virus/ 
bacterial typing $[15,16]$. However, as sequencing is cumbersome, expensive and less likely to detect low prevalence mutations (mutations consisting less than $30 \%$ of total populations) $[17,18]$, newer alternative techniques such as real time PCR and RCA are being employed $[9,15]$. To establish molecular assays, positive controls are a prerequisite to ascertain specificity and sensitivity $[19,20]$. However, in many cases it is difficult and cumbersome to acquire appropriate positive controls. It is thus, important to mention that although the commercial oligonucleotide synthesis can generate long oligonucleotides ( $\geq 150$ mers) and can serve as suitable controls, there are problems as the oligonucleotide length exceeds 100 nucleobases, the yield of desired products often becomes limited by side reactions and even modest inefficiencies within the stepwise chemical reactions can have large effects on the final yield [21].

Further, in the event of an influenza pandemic, drug resistant strains and their transmission could be clinically highly significant [1], meaning that sensitive and specific techniques are required for their early and clinically relevant detection. However, due to the low frequency of naturally occurring resistance mutations in influenza infected patients receiving NA inhibitor treatment, the highly pathogenetic nature of influenza A H5N1 strains, and the technical complexity and time consuming nature of generating of NA resistant strains in vitro, collection of all known resistance mutations as positive controls is challenging. Therefore, our approach utilizing the formation of oligonucleotide dimers between two commercially synthesised long single stranded DNA templates ( $\sim 95$ bases each), to generate $\sim 170$ bp double-stranded artificial DNA templates, containing resistance mutations is not only innovative, but a more molecularly feasible and durable. In this way, it offers significant advantages in synthesizing even longer double stranded DNA templates, which can be used as positive control templates in molecular diagnostics. In the present study, we have used these double stranded artificial DNA templates with all known genetic mutations associated with influenza A drug resistance as a positive control in the development of a ligase chain reaction (LCR) for detecting NA inhibitor drug resistance mutations in patient samples.

\section{Results}

Generation of ds DNA template from synthetic oligos

After 5 PCR reaction cycles, the products from paired synthetic long oligos were run on $2 \%$ agarose gel, stained with ethidium bromide and viewed under UV light. The dsDNA products were clearly visualized at the size 170 bp (Figure 1), demonstrating a successful production of long ds DNA templates from synthetic oligos.

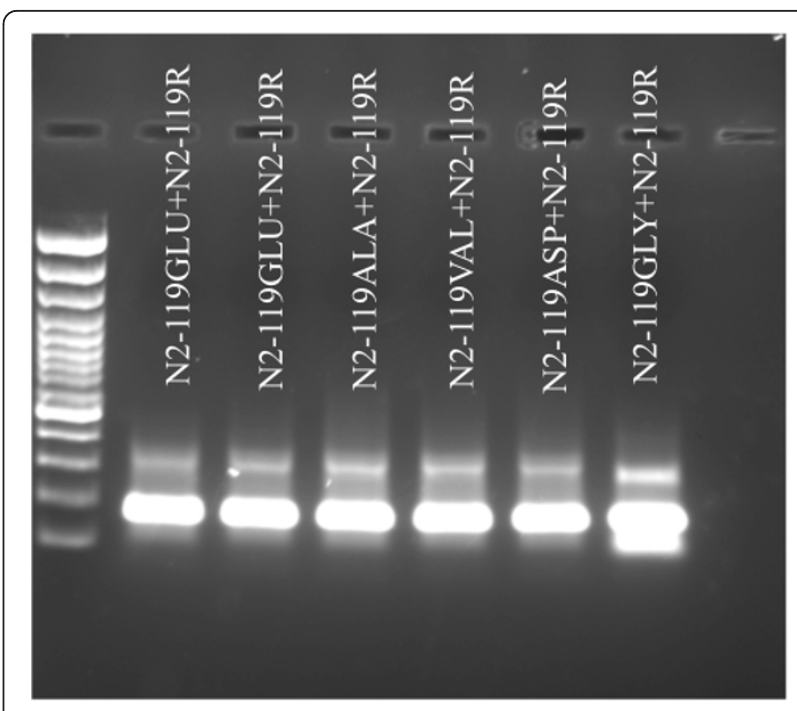

Figure 1 Generation of double stranded DNA templates using paired synthetic long oligos. After 5 cycles of $P C R$, the products were run on $2 \%$ agarose gel. The formation of $\sim 170 \mathrm{bp}$ of product can be clearly visualized.

\section{Cloning of DNA products and sequencing of positive clones}

The PCR products from each paired oligo were cloned and 5 positive clones were sequenced to validate the presence of desired mutations. Each sequence was aligned carefully and compared with the artificial template and reference strains. As expected, most sequences were identical to the artificial template design carrying resistance mutations (Table 1).

\section{Ligase Chain Reaction in the detection of resistance mutation}

Standard liner template containing resistance mutations (N1: His274Tyr, H5N1 His274Tyr, N2 Glu119Val, and N2 Ag292Lys) at various levels were prepared by mixing wild-type template with template containing resistance mutations. Each standard template was targeted by four independent probes specifically recognizing each of the resistance mutation. After 10 cycles of ligation reaction, even the presence of low-level of resistance mutation ( $1 \%)$ could be detected, as evidence by the formation of specific dsDNA molecules of about 80-90 bp (Figure 2).

\section{Discussion and Conclusions}

Although sequencing serves as a 'gold standard' for the detection of single nucleotide polymorphisms associated with drug resistance mutations or organism typing methods [15,16], alternative techniques are being employed $[9,15,22,23]$. One impediment to these techniques is the lack of availability of control templates carrying rare mutant variants/alleles or all relevant mutations, 
Table 1 List of artificial templates containing wild type and NA-resistance mutations.

\begin{tabular}{|c|c|c|c|}
\hline $\begin{array}{l}\text { Oligo } \\
\text { name }\end{array}$ & Oligo Sequence & $\begin{array}{c}\text { Location in reference } \\
\text { strains } \\
\end{array}$ & Description \\
\hline $\begin{array}{l}\mathrm{N} 1274 \mathrm{H} \\
95 \mathrm{nt}\end{array}$ & 5'TCGTACAAAATCTTCAAGATCGAAAAGGGAAAGGTTACTAAATCAATAGAGTTGAATGCACCCAATTTCATTATGAGGAATGTTCCTGTTACCC 3’ & H1N1 (CY12306.1, 763-857) & Wild Type \\
\hline $\begin{array}{l}\text { N1 274Y } \\
95 \mathrm{nt}\end{array}$ & 5'TCGTACAAAATCTTCAAGATCGAAAAGGGAAAGGTTACTAAATCAATAGAGTTGAATGCACCCAATTITATTATGAGGAATGTTCCTGTTACCC 3' & H1N1 (CY12306.1, 763-857) & $\begin{array}{l}\text { Resistance } \\
\text { mutation }\end{array}$ \\
\hline $\begin{array}{l}\text { N1 274R } \\
96 \mathrm{nt}\end{array}$ & 5'TTGATTAAAAGACACCCAAGGTCGATTTGAACCATGCCAGTTGTCCCTGCATACACACATCACTGTGCCAGTGTCTGGGTAACAGGAACATTCCTC3' & H1N1 (CY12306.1, 933-837) & Antisense oligo \\
\hline $\begin{array}{l}\mathrm{H} 5 \mathrm{~N} 1 \\
274 \mathrm{H} \\
95 \mathrm{nt}\end{array}$ & 5'TCACATAAGATCTTCAAAATGGAAAAAGGGAAAGTGGTTAAATCAGTCGAATTGGATGCTCCTAATTATCACTATGAGGAATGCTCCTGTTATCC3' & H5N1 (DQ493076, 694-788) & Wild Type \\
\hline $\begin{array}{l}\text { H5N1 274Y } \\
\text { 95nt }\end{array}$ & 5TCACATAAGATCTTCAAAATGGAAAAAGGGAAAGTGGTTAAATCAGTCGAATTGGATGCTCCTAATTATTACTATGAGGAATGCTCCTGTTATCC 3' & $\begin{array}{l}\text { H5N1 (DQ250165, } \\
\text { 694-788) }\end{array}$ & $\begin{array}{l}\text { Resistance } \\
\text { mutation }\end{array}$ \\
\hline $\begin{array}{l}\text { H5N1 274R } \\
\quad 96 n t\end{array}$ & 5'TTGATTGAAAGATACCCATGGCCGATTTGAGCCATGCCAATTATCCCTGCACACACATGTGATTTCGCCGGCATCAGGATAACAGGAGCATTCCTC3' & $\begin{array}{l}\text { H5N1 (DQ250165, } \\
\text { 864-768) }\end{array}$ & Antisense oligo \\
\hline $\begin{array}{l}\text { N2-119GLU } \\
\text { 90nt }\end{array}$ & 5'TCTAAGGACAATTCGATTCGGCTTTCCGCTGGTGGGGACATCTGGGTGACAAGAGARCCTTATGTGTCATGCGATCCTGACAAGTGTTATC 3’ & H3N2 (CY016653,305-395) & Wild Type \\
\hline $\begin{array}{l}\text { N2-119Val } \\
90 \mathrm{nt}\end{array}$ & 5'TCTAAGGACAATTCGATTCGGCTTTCCGCTGGTGGGGACATCTGGGTGACAAGAGTNCCTTATGTGTCATGCGATCCTGACAAGTGTTATC3’' & H3N2 (CY016653,305-395) & $\begin{array}{l}\text { Resistance } \\
\text { mutation }\end{array}$ \\
\hline $\begin{array}{l}\text { N2-119GLY } \\
\text { 90nt }\end{array}$ & 5'TCTAAGGACAATTCGATTCGGCTTTCCGCTGGTGGGGACATCTGGGTGACAAGAGGNCCTTATGTGTCATGCGATCCTGACAAGTGTTATC3' & $\begin{array}{l}\text { H3N2 (CY016653, } \\
\text { 305-395) }\end{array}$ & Polymorphisms \\
\hline $\begin{array}{l}\text { N2-119ALA } \\
\text { 90nt }\end{array}$ & 5TCTAAGGACAATTCGATTCGGCTTTCCGCTGGTGGGGACATCTGGGTGACAAGAGCNCCTTATGTGTCATGCGATCCTGACAAGTGTTATC3' & H3N2 (CY016653,305-395) & Polymorphisms \\
\hline $\begin{array}{l}\text { N2-119ASP } \\
\text { 90nt }\end{array}$ & 5'TCTAAGGACAATTCGATTCGGCTTTCCGCTGGTGGGGACATCTGGGTGACAAGAGAYCCTTATGTGTCATGCGATCCTGACAAGTGTTATC3' & $\begin{array}{l}\text { H3N2 (CY016653, } \\
\text { 305-395) }\end{array}$ & Polymorphisms \\
\hline $\begin{array}{l}\text { N2-119R } \\
98 \mathrm{nt}\end{array}$ & 5'CCGATAAGGGGTCCTATCATGTACTGTGTCATTTGAATGCCCGTTGTTTAGTGTTGTTCCCTGTCCAAGGGCAAATTGATAACACTTGTCAGGATCGC3' & $\begin{array}{l}\text { H3N2 (CY016653, } \\
472-375)\end{array}$ & Antisense oligo \\
\hline $\begin{array}{l}\text { N2-292ARG } \\
\text { 89nt }\end{array}$ & CAGCATGTCGAGGAGTGCTCCTGTTATCCTCGATATCCTGGTGTCAGATGTGTCTGCAGRGACAACTGGAAAGGCTCCAATAGGCCCAT3’ & $\begin{array}{l}\text { H3N2 (CY016653, } \\
\text { 821-909 }\end{array}$ & Wild Type \\
\hline $\begin{array}{l}\text { N2-292LYS } \\
\text { 89nt }\end{array}$ & 5'CAGCATGTCGAGGAGTGCTCCTGTTATCCTCGATATCCTGGTGTCAGATGTGTCTGCAARGACAACTGGAAAGGCTCCAATAGGCCCAT3' & $\begin{array}{l}\text { H3N2 (CY016653, } \\
\text { 821-909 }\end{array}$ & $\begin{array}{l}\text { Resistance } \\
\text { mutation }\end{array}$ \\
\hline $\begin{array}{l}\mathrm{N} 2-292 \mathrm{R} \\
99 \mathrm{nt}\end{array}$ & 5'TTTCTGGGTGTGTCTCCAACAAGTCCTGAGCACACATAACTGGAAACAATGCTATAATCCTTTACATTTATATCTACGATGGGCCTATTGGAGCCTTCC3' & $\begin{array}{c}\text { H3N2 (CY016653, } \\
\text { 987-889 }\end{array}$ & Antisense oligo \\
\hline
\end{tabular}

1: Synthetic templates containing the wild type, naturally occurring polymorphisms and resistance mutations. These genome locations were indicated in the table according to reference strains.

2: Antisense oligo (R) with complementary sequences at 3 ' end were also synthesised and the complementary sequence were shown as underline. 


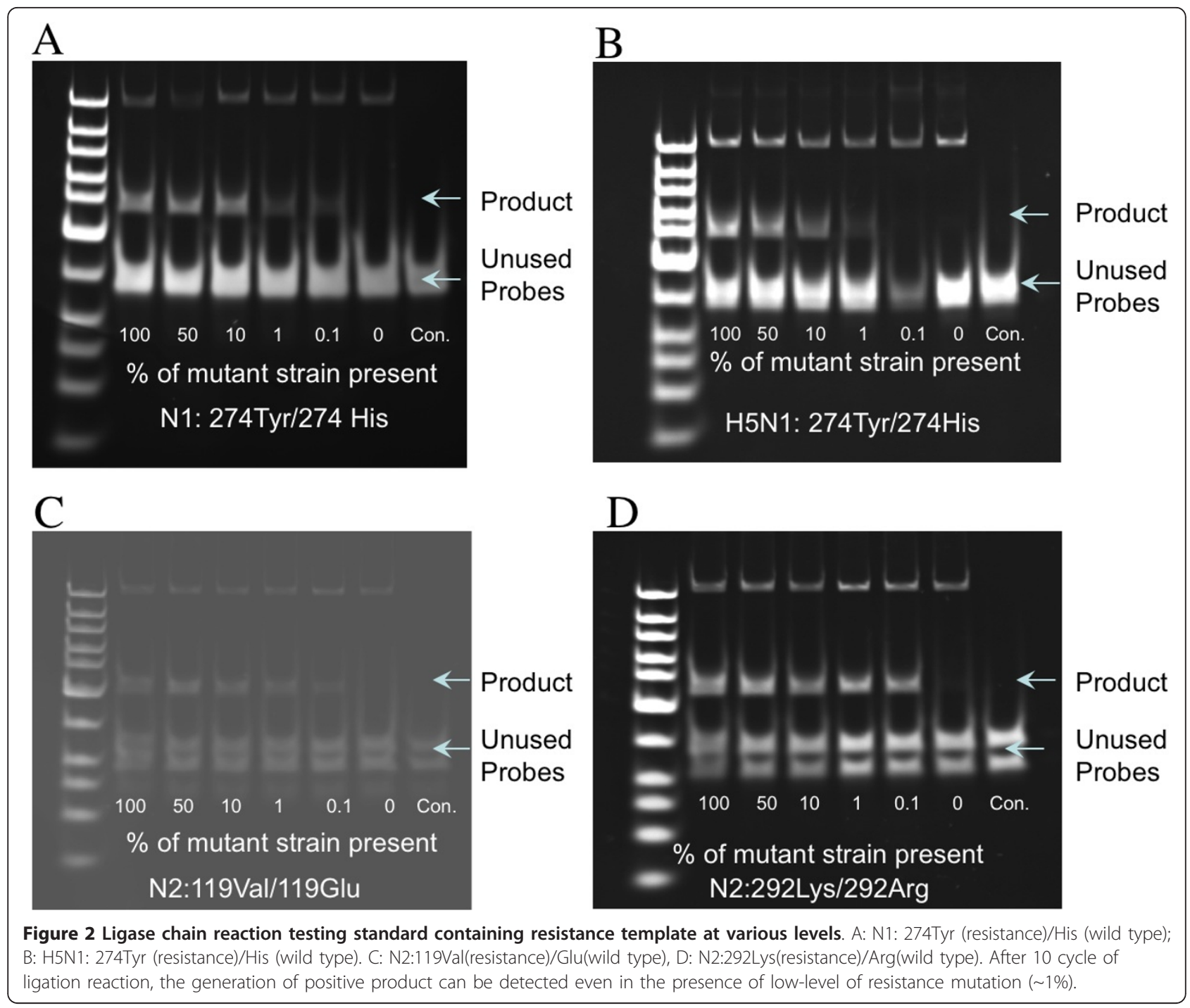

which can serve as positive controls in molecular or multiplex PCR assays simultaneously. In influenza studies, for example, several single nucleotide mutations are known to be associated with NA inhibitor resistance $[24,25]$. Many of these have been derived from different in vitro cultures, and currently naturally occurring NAI resistance appears to be uncommon in some strains. However, in the event of an influenza pandemic, the use of NAIs would dramatically increase and thus provide greater opportunities for resistance to emerge and spread. Therefore, rapid screening of these mutations, which would be of great benefit for patient treatment and the control of disease spread, should be available in a format that can facilitate simultaneous, rapid and bulk detection of drug resistance mutations. We were only able to source wild-type clinical isolates of influenza A H3N2; known resistant clinical strains were unavailable. Given that highly pathogenic avian influenza A H5N1 can only be handled in PC4 facilities, we had only limited access to influenza A H5N1 RNA. This was the main impediment to molecular assay development for the detection of drug resistance mutations. Thus, we endeavored to create artificial templates containing all previously identified single nucleotide mutations known for drug resistance within the neuraminidase gene of influenza virus. To address this issue, we produced long synthetic oligos containing known influenza A resistance mutations, and showed them to be effective as controls in LCR. Further, they can also serve as templates in real-time PCR and RCA [23,9].

The use of fully synthetic oligonucleotides as positive control material has been described recently [16]. However, there were limitations as to the length of the oligonucleotide, which was generally unable to reach more than 130 bases. In many situations, for instance in the case of drug resistance mutations, nucleotide 
substitutions are often scattered within the target genome and thus require larger fragments as standard templates. Here, we took a novel approach to creating long artificial templates by taking advantage of primerdimers, which are usually considered a common laboratory problem. The binding of two long artificially synthesized oligos generated a substrate for PCR and was used to create long double-stranded DNA molecules of about $170 \mathrm{bp}$. In most of the current diagnostic studies (especially based on real time PCR and RCA), 150-240 bp would be sufficient to serve a template controls. However, it is possible to use multiple long oligos to synthesize even longer double-stranded DNA and up to the full length of some important genes, if need be $[26,27]$, which is a significant advantage over any similar technologies existing currently.

The most significant advantage of using synthetic oligonucleotides is flexibility. Our approach has proven that the use of laboratory safe artificial templates can be a relatively cost effective, simple and efficient alternative to difficult to acquire material related to an infection or bioterrorism agent. The production of standard templates via the formation of oligo dimers and PCR using synthetic oligos does not require the use of viral or bacterial strains and oligos of any desired sequence can be made commercially. This approach may have the potential to produce a false-positive product due to contamination and therefore caution may be necessary. However, this problem can be solved by the introduction of exclusive restriction endonuclease digestion sites in the artificial template, which would allow for rapid confirmation of a false-positive result. Certain restriction endonuclease (SmaI and BamHI) can be used directly in the PCR buffer (without buffer exchange) and thus the PCR product from the control can be easily and rapidly distinguished from testing samples. In addition, the standard artificial template could be designed to be slightly smaller or larger in size, which would allow for direct visual identification of contamination with the synthetic control.

Overall, this technology may have wide ranging applications and may revolutionize molecular diagnostics and the use of multiplexing assays.

\section{Methods}

\section{Reference strains}

The reference strains of influenza A H1N1, H5N1 and H3N2 used in this study had Genebank accession numbers: CY12306.1, DQ493076, CY016653, respectively.

\section{Design and synthesis of long oligos}

Long single-stranded oligonucleotides of 89-98 bases were designed, each containing wild-type template or drug resistance mutations (Table 1). After synthesis, each oligonucleotide was PAGE purified (Sigma-Aldrich, Sydney, Australia). Paired long oligos were designed carrying $>20 \mathrm{bp}$ complementary sequences at their 3 ' ends.

\section{Generation of double-stranded DNA using long oligos and Taq polymerase}

20 pmol of each paired oligo was incubated with $2 \mathrm{U}$ of Taq DNA polymerase (Promega, Madison, USA), 1X reaction buffer, $2.5 \mathrm{mM} \mathrm{MgCl} 2$, and $0.4 \mathrm{mM}$ of dNTPs. The reaction mix was incubated at $94^{\circ} \mathrm{C}$ for 30 seconds followed by $55^{\circ} \mathrm{C}$ for 30 seconds and $72^{\circ} \mathrm{C}$ for $30 \mathrm{sec}-$ onds for 5 cycles. After the reaction, the end product was run on a $2 \%$ agarose gel and visualized under UV light with ethidium bromide staining.

During this process, the complementary sequences located at 3' end of the paired oligo would form a dimer and in the presence of DNA polymerase and dNTP, each oligo would extend its 3 ' end using the second oligo as a template (Figure 3).

\section{Cloning of the double-stranded DNA}

To ensure the purity of the standard template, the products were ligated into the pGEM-T Easy Vector System II (Promega, Madison, USA) and transformed into competent Escherichia coli JM109 cells, according to the manufacturer's protocol. Screening for the gene insert was performed by quick lysis at $95^{\circ} \mathrm{C}$ of the E. coli cells for 5 minutes, followed by PCR using $5 \mu \mathrm{l}$ of the cell lysate as previously described [28].

\section{Sequencing of cloned dsDNA and sequence analysis}

The plasmid DNA from positive clones was extracted using the QIAprep Spin Miniprep Kit according to the manufacturer's protocol (Qiagen, Melbourne, Australia). The inserts were sequenced using Applied Biosystems BigDye terminator chemistry version 3.1 (Foster City, CA, USA), on an ABI Prism 373 DNA sequencer. Sequences were identified using the FastA program group accessed through Biomanager (http://biomanager. info/).

Ligase Chain Reaction (LCR) in the detection of templates containing resistance mutation

Liner templates containing resistance and wild type were generated by PCR amplification of plasmid DNA, followed by purification using Millipore PCR purification plate (Millipore, Billerica, MA, USA). The linear PCR products were quantitated using spectrophotometer and dsDNA DNA copy number were estimated by DNA calculator (http://www.uri.edu/research/gsc/resources/ cndna.html). $5 \times 10^{11}$ copies of standard templates were used for testing the specificity of the LCR system. LCR probes targeting resistance template were designed (Table 2). Each template was targeted by four LCR 
Table 2 LCR probes for resistance mutations detection

\begin{tabular}{|c|c|}
\hline $\begin{array}{l}\text { Mutation } \\
\text { type }\end{array}$ & Probe sequences \\
\hline \multirow[t]{4}{*}{ N2-119VAL } & 5' AAGGGGACATCTGGGTGACAAGAGT 3' \\
\hline & 5' 'P-NCCTTATGTGTCATGCGATCCTGACAA 3' \\
\hline & 5' ACCTTGTCAGGATCGCATGACACATAAGGNA 3' \\
\hline & 5' P-CTCTTGTCACCCAGATGTCCCCAC 3' \\
\hline \multirow[t]{4}{*}{ N2-292LYS } & 5' ATGATATCCTGGTGTCAGATGTGTCTGCAA 3' \\
\hline & 5' P-RGACAACTGGAAAGGCTCCAATAGGC 3' \\
\hline & 5' CCCCTATTGGAGCCTTTCCAGTTGTCYT 3' \\
\hline & 5' P-TGCAGACACATCTGACACCAGGATATCGAG 3' \\
\hline \multirow[t]{4}{*}{ N1-274Y } & 5' TGTTACTAAATCAATAGAGTTGAATGCACCCAATTाT 3' \\
\hline & 5' P-ATTATGAGGAATGTTCCTGTTACCCACACAC 3' \\
\hline & 5' TTGTGTCTGGGTAACAGGAACATTCCTCATAATA 3' \\
\hline & $\begin{array}{l}\text { 5' P-AAAATTGGGTGCATTCAACTCTATTGATTTAGTAACC } \\
3^{\prime}\end{array}$ \\
\hline \multirow[t]{4}{*}{ H5N1-274Y } & 5' TCGGTTAAATCAGTCGAATTGGATGCTCCTAATTATT 3' \\
\hline & 5' P-ACTATGAGGAATGCTCCTGTTATCCTGATG 3' \\
\hline & 5' TTGCATCAGGATAACAGGAGCATTCCTCATAGTA 3' \\
\hline & 5' P-ATAATTAGGAGCATCCAATTCG 3' \\
\hline
\end{tabular}

a. P- indicates $5^{\prime}$ phosphorylation probes about 35-45nt with 2 of the probes having 5 ' end phosphorylation. Ligation of LCR probes to standard templates was carried out by mixing the standard template with $1 \mathrm{pmol}$ of each LCR probes, $2 \mathrm{U}$ of pfu DNA ligase (Stratagene, Integrated Sciences, Cedar Creek, TX, USA) in $20 \mathrm{mM}$ Tris- $\mathrm{HCl}$ (pH 7.5), $20 \mathrm{mM} \mathrm{KCl}, 10$ $\mathrm{mM} \mathrm{MgCl} 2$, 0.1\% Igepal, $0.01 \mathrm{mM}$ rATP, $1 \mathrm{mM}$ DTT with total reaction volume of $25 \mu \mathrm{l}$. Multiple cycle ligation was conducted to validate the specificity of the probe in recognizing its corresponding template. The reaction condition include one cycle of $5 \mathrm{~min}$ at $94^{\circ} \mathrm{C}$ to denature the dsDNA followed 10 cycles of by $94^{\circ} \mathrm{C} 30 \mathrm{~s}$ and 4 min ligation at $65^{\circ} \mathrm{C}$. The final product were run on 10\% TBE gel (Invitrogen, Mount Waverley VIC Australia) and visualized under UV light with ethidium bromide staining.

\section{List of abbreviations}

NAl: Neuraminidase inhibitor; PCR: polymerase chain reaction; RLB:reverse line blot hybridization; LCR: Ligase chain reaction; RCA: Rolling Circle Amplification. 


\section{Acknowledgements}

The authors gratefully acknowledge all colleagues who participated in this project. This work was funded by the NHMRC grant 408114, 633027 .

\section{Author details}

${ }^{1}$ Retroviral Genetics Laboratory, Centre for Virus Research, Westmead Millennium Institute, Westmead Hospital, The University of Sydney, Westmead, New South Wales 2145, Australia. ${ }^{2}$ Department of Infectious Diseases and Immunology, University of Sydney, Blackburn Building, 2006 NSW Australia. ${ }^{3}$ Centre for Infectious Diseases and Microbiology (CIDM), Institute of Clinical Pathology and Medical Research (ICPMR), Westmead, New South Wales 2145, Australia.

\section{Authors' contributions}

BW conceived, designed and conducted the study along with sequence search, data analyses and manuscript writing. MS assisted with the assay optimization, cloning, DNA sequencing. DD, AC and NS provided intellectual input and manuscript writing. All authors have read and approved the manuscript.

\section{Competing interests}

The authors declare that they have no competing interests.

Received: 19 January 2011 Accepted: 16 August 2011

Published: 16 August 2011

\section{References}

1. Ford SM, Grabenstein JD: Pandemics, avian influenza A (H5N1), and a strategy for pharmacists. Pharmacotherapy 2006, 26:312-322.

2. Thomas JK, Noppenberger J: Avian influenza: A review. American Journal of Health-System Pharmacy 2007, 64:149-165.

3. Octaviani CP, Ozawa M, Yamada S, Goto H, Kawaoka Y: High Level of Genetic Compatibility between Swine-Origin H1N1 and Highly Pathogenic Avian H5N1 Influenza Viruses. Journal of Virology 2010, 84:10918-10922.

4. Desheva JA, Lu XH, Rekstin AR, Rudenko LG, Swayne DE, Cox NJ, Katz JM, Klimov Al: Characterization of an influenza A H5N2 reassortant as a candidate for live-attenuated and inactivated vaccines against highly pathogenic H5N1 viruses with pandemic potential. Vaccine 2006, 24:6859-6866.

5. Qiao C, Tian G, Jiang Y, Li Y, Shi J, Yu K, Chen H: Vaccines developed for H5 highly pathogenic avian influenza in China. Ann N Y Acad Sci 2006, 1081:182-192.

6. Hayden F, Klimov A, Tashiro M, Hay A, Monto A, McKimm-Breschkin J, Macken C, Hampson A, Webster RG, Amyord M, Zambon M: Neuraminidase inhibitor susceptibility network position statement: antiviral resistance in influenza A/H5N1 viruses. Antiviral Therapy 2005 , 10:873-877.

7. Kiso M, Mitamura K, Sakai-Tagawa Y, Shiraishi K, Kawakami C, Kimura K, Hayden FG, Sugaya N, Kawaoka Y: Resistant influenza A viruses in children treated with oseltamivir: descriptive study. Lancet 2004, 364:759-765.

8. de Jong MD, Tran TT, Truong HK, Vo MH, Smith GJ, Nguyen VC, Bach VC, Phan TQ, Do QH, Guan Y, et al: Oseltamivir resistance during treatment of influenza A (H5N1) infection. N Engl J Med 2005, 353:2667-2672.

9. Wang B, Dwyer DE, Blyth CC, Soedjono M, Shi HJ, Kesson A, Ratnamohan M, McPhie K, Cunningham AL, Saksena NK: Detection of the rapid emergence of the $\mathrm{H} 275 \mathrm{Y}$ mutation associated with oseltamivir resistance in severe pandemic influenza virus A/H1N1 09 infections. Antiviral Research 2010, 87:16-21.

10. Kong FR, Gilbert GL: Multiplex PCR-based reverse line blot hybridization assay (mPCR/RLB) - a practical epidemiological and diagnostic tool. Nature Protocols 2006, 1:2668-2680.

11. Tong ZS, Kong FR, Wang B, Zeng XY, Gilbert GL: A practical method for subtyping of Streptococcus agalactiae serotype III, of human origin, using rolling circle amplification. Journal of Microbiological Methods 2007, 70:39-44.

12. Pourmand N, Diamond L, Garten R, Erickson JP, Kumm J, Donis RO, Davis RW: Rapid and highly informative diagnostic assay for H5N1 influenza viruses. PLoS One 2006, 1:e95.
13. Millar BC, XU JR, Moore JE: Molecular diagnostics of medically important bacterial infections. Current Issues in Molecular Biology 2007, 9:21-39.

14. Belak S: Molecular diagnosis of viral diseases, present trends and future aspects a view from the OIE collaborating centre for the application of polymerase chain reaction methods for diagnosis of viral diseases in veterinary medicine. Vaccine 2007, 25:5444-5452.

15. Benlloch S, Paya A, Alenda C, Bessa X, Andreu M, Jover R, Castells A, Llor X, Aranda Fl, Massuti B: Detection of BRAF V600E mutation in colorectal cancer: Comparison of automatic sequencing and real-time chemistry methodology. Journal of Molecular Diagnostics 2006, 8:540-543.

16. Costabile M, Quach A, Ferrante A: Molecular approaches in the diagnosis of primary immunodeficiency diseases. Hum Mutat 2006, 27:1163-1173.

17. Gunthard HF, Wong JK, Ignacio CC, Havlir DV, Richman DD: Comparative performance of high-density oligonucleotide sequencing and dideoxynucleotide sequencing of HIV type 1 pol from clinical samples. Aids Research and Human Retroviruses 1998, 14:869-876.

18. Palmer S, Boltz V, Maldarelli F, Kearney M, Halvas EK, Rock D, Falloon J, Davey RT, Dewar RL, Metcalf JA, et al: Selection and persistence of nonnucleoside reverse transcriptase inhibitor-resistant HIV-1 in patients starting and stopping non-nucleoside therapy. Aids 2006, 20:701-710.

19. Di Trani L, Bedini B, Donatelli I, Campitelli L, Chiappini B, De Marco MA, Delogu M, Buonavoglia C, Vaccari G: A sensitive one-step real-time PCR for detection of avian influenza viruses using a MGB probe and an internal positive control. Bmc Infectious Diseases 2006, 6.

20. Petrich A, Mahony J, Chong S, Broukhanski G, Gharabaghi F, Johnson G Louie L, Luinstra K, Willey B, Akhaven P, et al: Multicenter comparison of nucleic acid extraction methods for detection of severe acute respiratory syndrome coronavirus RNA in stool specimens. J Clin Microbiol 2006, 44:2681-2688.

21. LeProust EM, Peck BJ, Spirin K, McCuen HB, Moore B, Namsaraev E, Caruthers MH: Synthesis of high-quality libraries of long (150mer) oligonucleotides by a novel depurination controlled process. Nucleic Acids Research 2010, 38:2522-2540.

22. Wang B, Potter SJ, Lin YG, Cunningham AL, Dwyer DE, Su YL, Ma XJ, Hou YD, Saksena NK: Rapid and sensitive detection of severe acute respiratory syndrome coronavirus by rolling circle amplification. Journal of Clinical Microbiology 2005, 43:2339-2344.

23. Steain MC, Dwyer DE, Hurt AC, Kol C, Saksena NK, Cunningham AL, Wang B: Detection of influenza A H1N1 and H3N2 mutations conferring resistance to oseltamivir using rolling circle amplification. Antiviral Res 2009, 84:242-248.

24. Yen HL, Herlocher LM, Hoffmann E, Matrosovich MN, Monto AS, Webster RG, Govorkova EA: Neuraminidase inhibitor-resistant influenza viruses may differ substantially in fitness and transmissibility. Antimicrobial Agents and Chemotherapy 2005, 49:4075-4084

25. Monto AS, McKimm-Breschkin JL, Macken C, Hampson AW, Hay A Klimov A, Tashiro M, Webster RG, Aymard M, Hayden FG, Zambon M: Detection of influenza viruses resistant to neuraminidase inhibitors in global surveillance during the first 3 years of their use. Antimicrobial Agents and Chemotherapy 2006, 50:2395-2402.

26. Encke J, Putlitz JZ, Heintges T, Wands JR: Total chemical synthesis of the 3 ' untranslated region of the hepatitis $C$ virus with long oligodeoxynucleotides. Journal of Virological Methods 1998, 74:117-121.

27. Stemmer WP, Crameri A, Ha KD, Brennan TM, Heyneker HL: Single-step assembly of a gene and entire plasmid from large numbers of oligodeoxyribonucleotides. Gene 1995, 164:49-53.

28. Potter SJ, Lemey P, Achaz G, Chew CB, Vandamme AM, Dwyer DE, Saksena NK: HIV-1 compartmentalization in diverse leukocyte populations during antiretroviral therapy. J Leukoc Biol 2004, 76:562-570.

doi:10.1186/1743-422X-8-405

Cite this article as: Wang et al: Synthetic long oligonucleotides to generate artificial templates for use as positive controls in molecular assays: drug resistance mutations in influenza virus as an example. Virology Journal 2011 8:405. 